\title{
Rational Divisors in Rational Divisor Classes
}

\author{
N. Bruin ${ }^{\star 1}$ and E.V. Flynn ${ }^{\star 2}$ \\ 1 Department of Mathematics, Simon Fraser University \\ Burnaby, BC, Canada V5A 1S6 \\ nbruin@cecm.sfu.ca \\ 2 Mathematical Institute, 24-29 St. Giles, University of Oxford \\ Oxford OX1 3LB, United Kingdom \\ flynn@maths.ox.ac.uk
}

\begin{abstract}
We discuss the situation where a curve $\mathcal{C}$, defined over a number field $K$, has a known $K$-rational divisor class of degree 1 , and consider whether this class contains an actual $K$-rational divisor. When $\mathcal{C}$ has points everywhere locally, the local to global principle of the Brauer group gives the existence of such a divisor. In this situation, we give an alternative, more down to earth, approach, which indicates how to compute this divisor in certain situations. We also discuss examples where $\mathcal{C}$ does not have points everywhere locally, and where no such $K$-rational divisor is contained in the $K$-rational divisor class.
\end{abstract}

\section{Introduction}

The following result is typically proved as a direct consequence of the local to global principle of the Brauer group (see, for example, [2] or p.30 of [3]).

Lemma 1 Let $\mathcal{C}$ be a curve defined over a number field $K$ with points everywhere locally. Then any $K$-rational degree 1 divisor class $\mathcal{D}$ contains a $K$-rational divisor $D$.

Such divisor classes have relevance to the application of second descents [4], as well as the application of the Brauer-Manin obstruction to higher genus curves, where such a class $\mathcal{D}$ is used to obtain an embedding $P \mapsto[P]-\mathcal{D}$ from $\mathcal{C}(K)$ to $J(K)$, the Mordell-Weil group of the Jacobian. This embedding can sometimes be used to find information about $\mathcal{C}(K)$.

Our intention here is to describe, in a concise and explicit manner, how the problem of finding a rational divisor in a rational divisor class corresponds to finding a rational point on a certain algebraic variety. We give an example of how this description can be used in practice to find a rational divisor explicitly, given a rational divisor class.

* Partially supported by an NSERC grant.

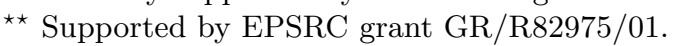




\section{The Brauer-Severi variety of a divisor class}

Let $\mathcal{C}$ be a curve defined over a field $K$ and let $\bar{K}$ be a separable closure of $K$. We say a divisor class $\mathcal{D} \in \operatorname{Pic}_{\mathcal{C}}(\bar{K})$ is rational if it is fixed under the action of $\operatorname{Gal}(\bar{K} / K)$. This means that for any divisor $D \in \mathcal{D}$ and $\sigma \in \operatorname{Gal}(\bar{K} / K)$, we have that ${ }^{\sigma} D$ and $D$ are linearly equivalent. In the language of Galois modules and Galois cohomology, we have

$$
\operatorname{Pic}_{\mathcal{C}}(\bar{K})^{K}=H^{0}\left(K, \operatorname{Pic}_{\mathcal{C}}(\bar{K})\right)=\left\{\mathcal{D} \in \operatorname{Pic}_{\mathcal{C}}(\bar{K}): \mathcal{D} \text { is a rational divisor class }\right\}
$$

This group is different from $\operatorname{Pic}_{\mathcal{C}}(K)$, which simply consists of the linear equivalence classes in $\operatorname{Div}_{\mathcal{C}}(K)$. There is an obvious embedding $\operatorname{Pic}_{\mathcal{C}}(K) \subset \operatorname{Pic}_{\mathcal{C}}(\bar{K})^{K}$ and we identify $\operatorname{Pic}_{\mathcal{C}}(K)$ with its image.

For $D \in \operatorname{Div}_{\mathcal{C}}(\bar{K})$, we adopt the standard notation

$$
\mathcal{L}(D)=\{f \in \bar{K}(\mathcal{C}):(f) \geq-D\}
$$

This is a finite dimensional vector space over $\bar{K}$. We write $l(D)$ for its dimension. The Riemann-Roch theorem asserts that, for any divisor $D \in \operatorname{Div}_{\mathcal{C}}(\bar{K})$ and any canonical divisor $\kappa$ of $\mathcal{C}$, we have $l(D)-l(\kappa-D)=\operatorname{deg} D-g+1$. Furthermore $l(D)$ only depends on the equivalence class of $D$ and we write $l([D])=l(D)$.

We write

$$
\mathcal{V}_{D}(\bar{K})=\mathbb{P} \mathcal{L}(D)
$$

for the complete linear system of $D$. Via the map $f \mapsto(f)+D$, we see that this set is in bijection with the set of effective divisors linearly equivalent to $D$ :

$$
\mathcal{V}_{D}(\bar{K}) \simeq \mathcal{V}_{[D]}(\bar{K}):=\left\{D^{\prime} \in \operatorname{Div}_{\mathcal{C}}(\bar{K}): D^{\prime} \geq 0 \text { and } D^{\prime} \sim D\right\}
$$

Let $\mathcal{D}$ be a rational divisor class with $l(\mathcal{D})>0$. Then $\mathcal{V}_{\mathcal{D}}(\bar{K})$ is fixed under $\operatorname{Gal}(\bar{K} / K)$ and has the structure of a Galois set. Generalizing the above notation, for an extension $L$ of $K$, we write $\mathcal{V}_{\mathcal{D}}(L)$ for the effective divisors of $\mathcal{C}$ defined over $L$ and in $\mathcal{D}$.

The functor $\mathcal{V}_{\mathcal{D}}$ is represented by a scheme over $K$, which is called the BrauerSeveri variety associated to $\mathcal{D}$.

It follows that the following are equivalent.

$-\mathcal{V}_{\mathcal{D}}(K) \neq \emptyset$

$-\mathcal{V}_{\mathcal{D}}(K) \simeq \mathbb{P}^{(l(\mathcal{D})-1)}(K)$

$-\mathcal{D}$ contains a rational divisor.

In some cases it is easy to see that the conditions above hold. For instance, if $\mathcal{D}$ is a rational divisor class with $l(\mathcal{D})=1$, then over $\bar{K}$, there is a unique effective divisor $D$ representing $\mathcal{D}$. Consequently, $D$ is fixed under $\operatorname{Gal}(\bar{K} / K)$ and therefore is itself rational.

If a curve has a rational point $P_{0}$ then the property above is sufficient to deduce that any rational divisor class contains rational divisors. We use that for any divisor $D$ and point $P$ the following inequalities hold:

$$
l(D) \leq l(D+P) \leq l(D)+1 .
$$


It follows from (4) that for any divisor class $\mathcal{D}$ there exists an integer $n$ such that $l\left(\mathcal{D}+\left[n P_{0}\right]\right)=1$. The argument above shows that the rational divisor class $\mathcal{D}+\left[n P_{0}\right]$ contains a rational divisor $D$ and therefore $D-n P_{0} \in \mathcal{D}$.

In particular, if a curve $\mathcal{C}$ over a number field $K$ has points everywhere locally and $l(\mathcal{D})>0$ then $\mathcal{V}_{\mathcal{D}}$ has rational points everywhere locally. Hence, Lemma 1 is equivalent to the assertion that the local-to-global principle applies to the Brauer-Severi varieties $\mathcal{V}_{\mathcal{D}}$. For $l(\mathcal{D})=2$, we have that $\mathcal{V}_{\mathcal{D}}$ is a curve of genus 0 , and the Hasse-Minkowski theorem confirms that such varieties obey a local-to-global principle.

In fact, without assuming local solvability of $\mathcal{C}$, the geometry of $\mathcal{V}_{\mathcal{D}}$ still allows deductions to be made about representability of divisor classes by rational divisors. For instance, any $\mathcal{D}$ with $l(\mathcal{D})=2$ is representable by a divisor over a quadratic extension of $K$, since any curve of genus 0 has quadratic points.

We now sketch how one can proceed, given a rational divisor class $\mathcal{D}$, to derive an explicit model of $\mathcal{V}_{\mathcal{D}}$ in such a way that finding a rational point on it allows the construction of a representing rational divisor. Suppose that $\mathcal{D}$ is represented by a divisor $D$ over some finite extension $L=K(\alpha)$ of $K$ of degree, say, $d$.

1. Determine a basis $f_{1}, \ldots, f_{l(D)} \in L(\mathcal{C})$ of $\mathcal{L}(D)$.

2. Over $L$, we have $\mathcal{V}_{\mathcal{D}} \simeq \mathbb{P}^{l(D)-1}$ via the inverse of the map

$$
\left(t_{1}: \cdots: t_{l(D)}\right) \mapsto D+\left(t_{1} f_{1}+\cdots+t_{l(D)} f_{l(D)}\right) .
$$

This establishes a model of $\mathcal{V}_{\mathcal{D}}$ over $L$, with $\left(t_{1}: \ldots: t_{l(D)}\right)$ as projective coordinates. Putting $t_{1}=1$ yields an affine chart $\left(t_{2}, \ldots, t_{l(D)}\right)$ of $\mathcal{V}_{\mathcal{D}}$ over $L$.

3. In order to descend our model of $\mathcal{V}_{\mathcal{D}}$ over $L$ to a model over $K$, we compute a representation of $D_{t}=D+\left(f_{1}+t_{2} f_{2}+\cdots t_{l(D)} f_{l(D)}\right) \in \operatorname{Div}_{\mathcal{C}}\left(L\left(t_{2}, \ldots, t_{l(D)}\right)\right)$ corresponding to the generic point $\left(1: t_{2}: \ldots: t_{l(D)}\right)$ on $\mathcal{V}_{\mathcal{D}}$ as a scheme over $L$.

Let $\mathcal{C}$ be given as a plane curve with coordinates $X$ and $Y$ in general position over $K$. The effective divisor $D_{t}$ can be described by the equations $g(X)=0, Y=h(X)$, with $g, h \in L\left(t_{2}, \ldots, t_{l(D)}\right)[X]$, with $g$ monic, $\operatorname{deg}(g)=\operatorname{deg}(D)$ and $\operatorname{deg}(h)=\operatorname{deg}(D)-1$, since we have taken $X$ and $Y$ such that degeneracies do not occur.

4. We substitute $t_{i}=\sum_{j=0}^{d-1} t_{i, j} \alpha^{j}$ and write

$$
g(X)=\sum_{k=0}^{d-1} g_{k}(X) \alpha^{j}, \text { where } g_{k}(X) \in K\left(\left\{t_{i, j}\right\}_{i, j}\right)[X]
$$

and similarly for $h(X)$. A point $\left(1: t_{1}: \cdots: t_{l(D)}\right)$ corresponds to a divisor over $K$ precisely when that divisor can be described by equations not involving $\alpha$. Thus, we are led to consider the equations obtained by insisting that $g_{k}(X)=h_{k}(X)=0$ for $k=1, \ldots, d-1$ as polynomials in $X$. Those equations define an affine chart of $\mathcal{V}_{\mathcal{D}}$ over $K$, with coordinates $t_{i, j}$ over $K$. To get a model of $\mathcal{V}_{\mathcal{D}}$, one can take the projective closure. 
5. If one finds a point $\left(t_{i, j}\right)$, one can reconstruct $g, h$ from these values and obtain a description of a $K$-rational divisor in $\mathcal{D}$. Equivalently, one can reconstruct $\left(1: t_{2}: \cdots: t_{l(D)}\right)$ and thus obtain a $K$-rational specialization of $D_{t}$.

Of course, the procedure described above only applies to divisor classes satisfying $l(\mathcal{D})>0$. In general, one should select some divisor $D_{0}$ over $K$, preferrably of minimal positive degree, and an integer $n$ such that $l\left(\mathcal{D}+\left[n D_{0}\right]\right)$ is minimally positive. One can then apply the procedure to that divisor class and derive a suitable representative of $\mathcal{D}$ from the result, or conclude that none exists.

\section{Finding a rational divisor on a curve of genus 2}

We will give an example of this for a genus 2 curve

$$
\mathcal{C}: Y^{2}=f_{6} X^{6}+f_{5} X^{5}+\cdots+f_{0}=F(X), \text { with } F(X) \in K[X]
$$

and a rational divisor class $\mathcal{B}$ of degree 3 , represented by an effective divisor defined over a quadratic extension of $K$.

We assume that $\mathcal{B}$ is a rational divisor class with $\mathcal{B}=\left[P_{1}+P_{2}+P_{3}\right]$ and $P_{1}, P_{2}, P_{3} \in \mathcal{C}(K(\sqrt{d}))$, where the $P_{i}$ are not all Weierstrass points. It follows that $l(\mathcal{B})=2$. We can arrive at an equation for $\mathcal{V}_{\mathcal{B}}$ in the by following the outline in Section 2. We will give an account that can be read independently, but point out the correspondences with the general algorithm.

Let $G_{t}(x) \in K(\sqrt{d})[t][x]$ be the cubic in $x$ such that $y=G_{t}(x)$ passes through the points $P_{1}, P_{2}, P_{3}$ for all values of $t$. For any value $t \in K(\sqrt{d})$ we have

$$
\frac{\left(x-x_{1}\right)\left(x-x_{2}\right)\left(x-x_{3}\right)}{y-G_{t}(x)} \in \mathcal{L}(\mathcal{B}) .
$$

This allows us to write down a basis of $\mathcal{L}(\mathcal{B})$ for Step 1 in Section 2. We find $f_{1}=1, f_{2}=\frac{\left(x-x_{1}\right)\left(x-x_{2}\right)\left(x-x_{3}\right)}{y-G_{0}(x)}$. We get the coordinates $(1: t)$ on $\mathcal{L}(\mathcal{B})$.

For any value of $t$, we have that the identity

$$
\{y=G(x)\} \cap \mathcal{C}=P_{1}+P_{2}+P_{3}+\left(x_{1}, y_{1}\right)+\left(x_{2}, y_{2}\right)+\left(x_{3}, y_{3}\right) \simeq 3 \mathcal{O},
$$

where $\mathcal{O}$ is any canonical divisor ${ }^{3}$ of $\mathcal{C}$. The divisor $\left(x_{1}, y_{1}\right)+\left(x_{2}, y_{2}\right)+\left(x_{3}, y_{3}\right)$ can be described by $\left\{C_{t}(x)=0, y=H_{t}(x)\right\}$, where $C_{t}(x), H_{t}(x) \in K(\sqrt{d})[t][x]$ are a monic cubic and a quadratic in $x$ respectively. This is the required description for Step 3 of Section 2 .

Substituting $t=t_{1}+\sqrt{d} t_{2}$, we get that the coefficients $c_{i}$ of $C_{t_{1}+\sqrt{d} t_{2}}$ are of the form $c_{i}=c_{i, 0}\left(t_{1}, t_{2}\right)+\sqrt{d} c_{i, 1}\left(t_{1}, t_{2}\right)$, with $c_{i, j} \in K\left[t_{1}, t_{2}\right]$, and similarly for $H_{t}$. Setting the $\sqrt{d}$-components to 0 yields 6 equations in $t_{1}, t_{2}$ over $K$, which

\footnotetext{
${ }^{3}$ The divisor $\mathcal{O}=\infty^{+}+\infty^{-}$, consisting of the intersection of $\mathcal{C}$ with $X=\infty$, is a popular choice among people computing with curves of genus 2 .
} 
describe the genus 0 curve $\mathcal{V}_{\mathcal{B}}$. This corresponds to Step 4 of Section 2, where we put $t_{2}=t_{2,1}+\sqrt{d} t_{2,2}$.

If $\mathcal{C}$ has points everywhere locally, then Lemma 1 asserts that $\mathcal{V}_{\mathcal{B}}$ has a rational point. Let $C_{t_{0}}(x), H_{t_{0}}(x)$ correspond to such a point. Then the divisor class $\mathcal{B}$ is represented by the rational divisor $B=3 \mathcal{O}-\left\{C_{t_{0}}(x)=0, y=H_{t_{0}}(x)\right\}$. This corresponds to Step 5 of Section 2.

Of course, since there exists $\mathcal{O}$, which is of degree 2 and defined over $K$, the above can be applied to the rational divisor class $\mathcal{D}=\left[P_{1}+P_{2}+P_{3}-\mathcal{O}\right]$ of degree 1 , which is represented by the rational divisor $D=B-\mathcal{O}$.

We illustrate the above ideas with a detailed worked example. We first observe how a $K$-rational divisor class can arise naturally, in such a way that the contained $K$-rational divisor is not immediately apparent.

Let $\mathcal{C}$ be the genus 2 curve, defined over $\mathbb{Q}$,

$$
\mathcal{C}: Y^{2}=F(X)=-X^{6}-X^{5}-2 X^{4}-2 X^{3}+X^{2}-2 X+2,
$$

which is easily checked to have points in $\mathbb{R}$ and every $\mathbb{Q}_{p}$. One can perform a 2-descent on the Mordell-Weil group $\mathcal{J}(\mathbb{Q})$ of the Jacobian, as described in [6], using the map

$$
\mu: \mathcal{J}(\mathbb{Q}) \rightarrow \mathbb{Q}(\theta)^{*} / \mathbb{Q}^{*}\left(\mathbb{Q}(\theta)^{*}\right)^{2}:\left[\sum\left(x_{i}, y_{i}\right)\right] \mapsto \prod\left(x_{i}-\theta\right),
$$

where $\theta$ is a root of $F(X)$. One of the steps of the 2-descent on $\mathcal{J}(\mathbb{Q})$ is the computation of the kernel of $\mu$, generated by $2 \mathcal{J}(\mathbb{Q})$ and $\left[P_{1}+P_{1}^{\prime}-\mathcal{O}\right]$, where $P_{1}=\left(\frac{1}{2}+\frac{1}{5} \alpha, \frac{7}{40}+\frac{12}{25} \alpha\right)$ and $\alpha=\sqrt{-55}$, with $P_{1}^{\prime}$ denoting the conjugate of $P_{1}$ with respect to $\mathbb{Q}(\alpha) / \mathbb{Q}$. Let $P_{1}^{-}$be the hyperelliptic involute of $P_{1}$. Then general properties of $\mu$ (see [6] or p.55 of [3]) guarantee that $\left[P_{1}^{-}+P_{1}^{\prime}-\mathcal{O}\right] \in 2 \mathcal{J}(\mathbb{Q}(\alpha)$ ), and computing preimages under the multiplication by 2 map on $\mathcal{J}(\mathbb{Q}(\alpha))$, one finds $\mathcal{D}_{1}=[(1+\sqrt{-5}, 2 \alpha)+(1-\sqrt{-5}, 2 \alpha)-\mathcal{O}]$ which satisfies $\left[P_{1}^{-}+P_{1}^{\prime}-\mathcal{O}\right]=2 \mathcal{D}_{1}$. Clearly $\mathcal{D}_{1}^{\prime}=-\mathcal{D}_{1}$, since conjugation merely negates the $y$-coordinates. Let

$$
\begin{aligned}
P_{1} & =\left(\frac{1}{2}+\frac{1}{5} \alpha, \frac{7}{40}+\frac{12}{25} \alpha\right), P_{2}=(1+\sqrt{-5}, 2 \alpha), P_{3}=(1-\sqrt{-5}, 2 \alpha), \\
\mathcal{D} & =\left[P_{1}\right]+\mathcal{D}_{1}=\left[P_{1}+P_{2}+P_{3}-\mathcal{O}\right], \text { where } \alpha=\sqrt{-55} .
\end{aligned}
$$

Then

$\mathcal{D}^{\prime}=\left[P_{1}^{\prime}\right]+\mathcal{D}_{1}^{\prime}=\left[P_{1}^{\prime}\right]-\mathcal{D}_{1}=\left[P_{1}^{\prime}\right]-2 \mathcal{D}_{1}+\mathcal{D}_{1}=\left[P_{1}^{\prime}\right]-\left[P_{1}^{-}+P_{1}^{\prime}-\mathcal{O}\right]+\mathcal{D}_{1}=\mathcal{D}$,

so that $\mathcal{D}$ is defined over $\mathbb{Q}$. We now have a naturally occurring divisor class $\mathcal{D}$ of degree 1 , which is defined over $\mathbb{Q}$, but whose naturally occurring representative $P_{1}+P_{2}+P_{3}-\mathcal{O}$ is not itself defined over $\mathbb{Q}$. This is a common outcome of an application of 2-descent on $\mathcal{J}(\mathbb{Q})$ for genus 2 curves. Note that such $\mathcal{D}$ are of some interest, as they allow an embedding of $\mathcal{C}(\mathbb{Q})$ into $\mathcal{J}(\mathbb{Q})$ via the map $P \mapsto[P]-\mathcal{D}$, even when no obvious member of $\mathcal{C}(\mathbb{Q})$ is available.

Since our curve has points everywhere locally, we know that $\mathcal{D}$ does contain an actual $\mathbb{Q}$-rational divisor. We now illustrate how this can be found in practice. One first finds the general $Y=G(X)$, through $P_{1}, P_{2}, P_{3}$, where $G(X)$ is cubic 
in $X$, and where there is a free parameter $t$, since we have one less than the number of points required to define the cubic uniquely. This parametrized family of cubics is

$$
\begin{aligned}
G(X)= & t X^{3}+\left(\frac{3}{2}-\frac{5 t}{2}-\frac{2 \alpha}{5}-\frac{t \alpha}{5}\right) X^{2} \\
& +\left(-3+7 t+\frac{4 \alpha}{5}+\frac{2 t \alpha}{5}\right) X+9-3 t-\frac{2 \alpha}{5}-\frac{6 t \alpha}{5}
\end{aligned}
$$

Computing $G(X)^{2}-F(X)$, where $F(X)$ is as in (7), and removing the cubic factor $\left(X-X\left(P_{1}\right)\right)\left(X-X\left(P_{2}\right)\right)\left(X-X\left(P_{3}\right)\right)$ leaves the residual cubic

$$
\begin{aligned}
C(X)= & 10\left(1+t^{2}\right) X^{3}+\left(35+2 \alpha+30 t-8 t \alpha-25 t^{2}-2 t^{2} \alpha\right) X^{2} \\
& +\left(-50-4 \alpha-60 t+16 t \alpha+70 t^{2}+4 t^{2} \alpha\right) X \\
& +30+12 \alpha+180 t-8 t \alpha-30 t^{2}-12 t^{2} \alpha
\end{aligned}
$$

Let $\left(x_{1}, y_{1}\right)+\left(x_{2}, y_{2}\right)+\left(x_{3}, y_{3}\right)-\mathcal{O} \in \mathcal{D}$. Then $x_{1}, x_{2}, x_{3}$ are the roots of $C(X)$, for some choice of $t \in \mathbb{Q}(\alpha)$. Furthermore, the $y_{i}=-G\left(x_{i}\right)$, where $G(X)$ is as in (10). We now compute the quadratic $Y=H(X)$ which passes through $\left(x_{1},-G\left(x_{1}\right)\right),\left(x_{2},-G\left(x_{2}\right)\right),\left(x_{3},-G\left(x_{3}\right)\right)$, namely

$$
\begin{aligned}
H(X)= & \left(\left(15-60 t-15 t^{2}-4 \alpha-4 t \alpha+4 t^{2} \alpha\right) X^{2}\right. \\
& +\left(-30+120 t+30 t^{2}+8 \alpha+8 t \alpha-8 t^{2} \alpha\right) X \\
& \left.+90-60 t-90 t^{2}-4 \alpha-24 t \alpha+4 t^{2} \alpha\right) /\left(10+10 t^{2}\right)
\end{aligned}
$$

We have now parametrized divisors of the form $\left(x_{1}, y_{1}\right)+\left(x_{2}, y_{2}\right)+\left(x_{3}, y_{3}\right)-\mathcal{O}$ which are in $\mathcal{D}$. Our requirement for $\left(x_{1}, y_{1}\right)+\left(x_{2}, y_{2}\right)+\left(x_{3}, y_{3}\right)-\mathcal{O}$ to be Q-rational is the same as requiring that the ratios of the coefficients of $C(x)$ are $\mathbb{Q}$-rational (giving six polynomials in $t$, which can be reduced to three polynomials on the assumption that a given cofficient of $C(x)$ is nonzero; however, we shall prefer to write out all six polynomials in full), and that the actual coefficients of $H(x)$ are also $\mathbb{Q}$-rational (giving three polynomials in $t$ ). This gives in total nine polynomials in $t$ total that must be $\mathbb{Q}$-rational. Let $t=t_{1}+t_{2} \alpha$. Then on computing the coefficients on $\alpha$ in our nine expressions, we find nine quartics in $t_{1}, t_{2}$, all with a common factor of $t_{1}^{2}+55 t_{2}^{2}+15 t_{2}+1$. Then

$$
t_{1}^{2}+55 t_{2}^{2}+15 t_{2}+1=0
$$

is our desired curve of genus 0 , which has points everywhere locally, and hence globally. The solution of smallest height is $t_{1}=1 / 7, t_{2}=-1 / 7$, corresponding to $t=1 / 7-\alpha / 7$. Substituting this into $C(X)$ and $H(X)$, and removing from $C(X)$ a factor of $-5(5+2 \alpha) / 49$ (permissible, since the roots of $C(X)$ are unaffected), gives

$$
C(X)=2 X^{3}+X^{2}+2 X+2, \quad H(X)=-\frac{1}{2} X^{2}+X-1 .
$$

These $C(X), H(X)$ define $\left(x_{1}, y_{1}\right)+\left(x_{2}, y_{2}\right)+\left(x_{3}, y_{3}\right)-\mathcal{O}$, which is our desired $\mathbb{Q}$-rational divisor in our given $\mathbb{Q}$-rational divisor class $\mathcal{D}$. We summarize the above as follows. 
Example 1 Let $\mathcal{C}$ be the curve (7) and $\mathcal{D}$ be the $\mathbb{Q}$-rational divisor class in (9). Then $\mathcal{D}$ contains the $\mathbb{Q}$-rational divisor $\left(x_{1}, y_{1}\right)+\left(x_{2}, y_{2}\right)+\left(x_{3}, y_{3}\right)-\mathcal{O}$, where $x_{1}, x_{2}, x_{3}$ are the roots of $C(X)$ and $y_{i}=H\left(x_{i}\right)$ for $i=1,2,3$, with $C(X), H(X)$ as in (14).

We have written a Maple file at [1] which performs the above computation for any curve of genus 2 .

\section{Examples where the rational divisor class contains no rational divisor}

Suppose now that our genus 2 curve is defined over a number field $K$ and is of the form

$$
\begin{aligned}
& \mathcal{H}: Y^{2}=k F_{1}(X) F_{2}(X), \text { with } F_{1}(X)=a_{3} X^{3}+a_{2} X^{2}+a_{1} X+a_{0}, \\
& k \in K^{*}, \text { each } a_{i}=g_{i}+h_{i} \sqrt{d} \in K(\sqrt{d}), \text { and } F_{1}(X), F_{2}(X) \text { conjugate. }
\end{aligned}
$$

We shall also assume that $F_{1}(X)$ is not defined over $K$. Let $e_{1}, e_{2}, e_{3}$ denote the roots of $F_{1}(X)$. This is another situation where we have a naturally occurring degree 1 divisor class $\mathcal{D}=\left[\left(e_{1}, 0\right)+\left(e_{2}, 0\right)+\left(e_{3}, 0\right)-\mathcal{O}\right]$ which is defined over $K$, even though the given representative is defined over $K(\sqrt{d})$ and not generally over $K$. If $\mathcal{H}$ has points everywhere locally, we know that $\mathcal{D}$ must contain a divisor defined over $K$, but we do not make that assumption in this section. The parametrized familiy of cubics through $\left(e_{1}, 0\right),\left(e_{2}, 0\right),\left(e_{3}, 0\right)$ is simply $Y=G(X)=t F_{1}(X)$, where $t=t_{1}+t_{2} \sqrt{d}$ and $t_{1}, t_{2}$ are $K$-rational parameters. Replacing $Y$ by $t F_{1}(X)$ in $Y^{2}-k F_{1}(X) F_{2}(X)$ and removing the known factor $F_{1}(X)$ gives the residual cubic $C(X)=t^{2} F_{1}(X)-k F_{2}(X)$. Let $\left(x_{1}, y_{1}\right)+\left(x_{2}, y_{2}\right)+\left(x_{3}, y_{3}\right)-\mathcal{O} \in \mathcal{D}$. Then $x_{1}, x_{2}, x_{3}$ are the roots of $C(X)$, for some choice of $t \in K(\sqrt{d})$. Furthermore, each $y_{i}=-G\left(x_{i}\right)$; we compute the quadratic $Y=H(X)$ passing through the $\left(x_{i},-G\left(x_{i}\right)\right)$, namely

$$
\begin{aligned}
H(X) & =2 k \ell\left(\left(g_{2} h_{3}-g_{3} h_{2}\right) X^{2}+\left(g_{1} h_{3}-g_{3} h_{1}\right) X+g_{0} h_{3}-g_{3} h_{0}\right), \text { where } \\
\ell & =\left(-k+t_{2}^{2} d-t_{1}^{2}\right)\left(g_{3} t_{2}+h_{3} t_{1}\right) d-\left(k+t_{2}^{2} d-t_{1}^{2}\right)\left(g_{3} t_{1}+h_{3} t_{2} d\right) \sqrt{d} .
\end{aligned}
$$

Now, suppose that $\left(x_{1}, y_{1}\right)+\left(x_{2}, y_{2}\right)+\left(x_{3}, y_{3}\right)-\mathcal{O}$ is defined over $K$. Then the ratios of coefficients of $C(X)$ must be in $K$, giving the six equations

$$
\left(k+t_{2}^{2} d-t_{1}^{2}\right)\left(-k+t_{2}^{2} d-t_{1}^{2}\right)\left(g_{i} h_{j}-g_{j} h_{i}\right)=0, \text { for }\{i, j\} \subset\{0,1,2,3\}, i<j .
$$

Furthermore, the coefficients of $H(X)$ must be in $K$, giving the three equations

$$
\left(k+t_{2}^{2} d-t_{1}^{2}\right)\left(g_{3} t_{1}+h_{3} t_{2} d\right)\left(g_{i} h_{3}-g_{3} h_{i}\right)=0, \text { for } i=0,1,2 .
$$

Inspecting (17), we note that we cannot have all $g_{i} h_{j}-g_{j} h_{i}=0$, since then our original curve $\mathcal{H}$ would have zero discriminant. So, one of the first two factors in (17) must be 0 , giving that $\operatorname{Norm}(t)= \pm k$. If $\operatorname{Norm}(t)=-k$ then a similar argument (we have placed the details in the file [1]) applied to the equations (18) 
shows that either $\mathcal{H}$ has zero discriminant (which is not permitted) or that $F_{1}, F_{2}$ are each defined over $K$ (which is also not permitted). In summary, if $\mathcal{D}$ contains a $K$-rational divisor then we must have $\operatorname{Norm}(t)=k$ for some $t$.

Conversely, if $\operatorname{Norm}(t)=k$ for some $t \in K(\sqrt{d})$, then the $K$-rational divisor $\left(x_{1}, y_{1}\right)+\left(x_{2}, y_{2}\right)+\left(x_{3}, y_{3}\right)-\mathcal{O}$ in $\mathcal{D}$ is defined by

$$
C_{0}(X)=t F_{1}-t^{\prime} F_{2}(X), \quad H_{0}(X)=-4 k^{2} d\left(g_{3} t_{2}+h_{3} t_{1}\right) \sum_{i=0}^{2}\left(g_{i} h_{3}-g_{3} h_{i}\right) X^{i} .
$$

We summarize this as follows.

Lemma 2 Let $\mathcal{H}: Y^{2}=k F_{1}(X) F_{2}(X)$ be a curve of genus 2 of the type (15), defined over a number field $K$, where $F_{1}(X)$ is defined over $K(\sqrt{d})$ and not over $K$. Let $\mathcal{D}$ be the $K$-rational divisor class $\left[\left(e_{1}, 0\right)+\left(e_{2}, 0\right)+\left(e_{3}, 0\right)-\mathcal{O}\right]$, where $e_{1}, e_{2}, e_{3}$ are the roots of $F_{1}(X)$. Then $\mathcal{D}$ contains a $K$-rational divisor if and only if Norm $(t)=k$ for some $t \in K(\sqrt{d})$, in which case the required divisor is $\left(x_{1}, y_{1}\right)+\left(x_{2}, y_{2}\right)+\left(x_{3}, y_{3}\right)-\mathcal{O}$, where $x_{1}, x_{2}, x_{3}$ are the roots of $C_{0}(X)$ and each $y_{i}=H_{0}\left(x_{i}\right)$, with $C_{0}, H_{0}$ as in (19).

In this situation, our genus 0 curve is simply the curve $t_{1}^{2}-d t_{2}^{2}=k$. Of course, any choice of $K, d, k$ (such as $\mathbb{Q}, 2,5$ ), where $k$ is not a norm in $K(\sqrt{d}$ ), will give an example where $\mathcal{D}$ does not contain a $K$-rational divisor.

\section{References}

1. N. Bruin and E.V. Flynn. Maple programs for computing rational divisors in rational divisor classes. Available at www.maths.ox.ac.uk/ flynn/genus2/maple/ratdiv

2. D. Coray and C. Manoil. On large Picard groups and the Hasse principle for curves and K3 surfaces. Acta. Arith., LXXVI.2 (1996), 165-189.

3. J.W.S. Cassels and E.V. Flynn. Prolegomena to a Middlebrow Arithmetic of Curves of Genus 2. LMS-LNS 230. Cambridge University Press, Cambridge, 1996.

4. J.W.S. Cassels. Second descents for elliptic curves. J. reine angew. Math. 494 (1998), 101-127.

5. V. Scharaschkin. Local Global Problems and the Brauer-Manin Obstruction. PhD Thesis, University of Michigan, 1999.

6. M. Stoll. Implementing 2-descent for Jacobians of hyperelliptic curves. Acta Arith. 98 (2001), 245-277. 